\title{
Effect of aspect ratio on entropy generation in a rectangular cavity with differentially heated vertical walls is
}

\author{
Gamze Gediz Ilis ${ }^{a}$, Moghtada Mobedi ${ }^{a}$, Bengt Sunden ${ }^{\mathrm{b}, *}$ \\ ${ }^{a}$ Department of Mechanical Engineering, Izmir Institute of Technology, 35430, Urla, Izmir, Turkey \\ ${ }^{\mathrm{b}}$ Division of Heat Transfer, Lund Institute of Technology, Lund, Sweden
}

Available online 26 March 2008

\begin{abstract}
In the present study, entropy generation in rectangular cavities with the same area but different aspect ratios is numerically investigated. The vertical walls of the cavities are at different constant temperatures while the horizontal walls are adiabatic. Heat transfer between vertical walls occurs by laminar natural convection. Based on the obtained dimensionless velocity and temperature values, the distributions of local entropy generation due to heat transfer and fluid friction, the local Bejan number and local entropy generation number are determined and related maps are plotted. The variation of the total entropy generation and average Bejan number for the whole cavity volume at different aspect ratios for different values of the Rayleigh number and irreversibility distribution ratio are also evaluated. It is found that for a cavity with high value of Rayleigh number (i.e., $R a=10^{5}$ ), the total entropy generation due to fluid friction and total entropy generation number increase with increasing aspect ratio, attain a maximum and then decrease. The present results are compared with reported solutions and excellent agreement is observed. The study is performed for $10^{2}<R a<10^{5}, 10^{-4}<\phi<10^{-2}$, and $\operatorname{Pr}=0.7$.
\end{abstract}

(C) 2008 Elsevier Ltd. All rights reserved.

Keywords: Natural convection; Entropy generation; Bejan number

\section{Introduction}

Natural convection heat transfer in enclosures has recently been an important topic due to its wide applications in energy storage systems, electronic cooling devices, heating and cooling of buildings etc. Entropy is employed as a key parameter for evaluation of quality in engineering applications. The second law of thermodynamics has been applied to cavity problems to determine entropy generations due to heat and flow transport in the cavity and consequently minimize the entropy generation.

Dagtekin et al. [1] dealt with the prediction of entropy generation of natural convection in a $\Gamma$-shaped enclosure. They found that the main entropy generation is formed due to heat transfer for $R a<10^{5}$, the contribution due to fluid friction becomes stronger for $R a>10^{5}$. The transient state of entropy generation for laminar natural convection in a square cavity with heated vertical walls was numerically solved by Magherbi et al.

\footnotetext{
is Communicated by J.W. Rose and A. Briggs.

* Corresponding author.

E-mail address: bengt.sunden@energy.lth.se (B. Sunden).
}

[2]. Yilbas et al. [3] studied the natural convection and entropy generation in a square cavity with differential top and bottom wall temperatures. For the considered cavity, the total entropy generation increases with increasing wall temperature which means it becomes almost optimum for a certain Rayleigh number. Erbay et al. [4, 5] studied entropy generation during transient laminar natural convection in a square enclosure being heated either completely or partially from the left side wall and cooled from the opposite side wall. It is found that the active sides in the completely heated case are at the left bottom corner of the heated wall and right top corner of the cooled wall at the same magnitude. The optimization in an inclined square enclosure for minimum entropy generation was analyzed by Baytas [6]. Based on that study, the local heat transfer irreversibility and the local fluid friction irreversibility change by the inclination angle and the minimum entropy generation depends considerably on the inclination. Numerical prediction of local and total entropy generation rates for natural convection of air in a vertical channel symmetrically heated with a uniform heat flux was studied by Andreozzi et al. [7]. Mahmud and Islam [8] numerically investigated laminar free convection and entropy generation 


$\begin{array}{ll}\text { Nomenclature } \\ \mathrm{AR} & \text { Aspect ratio } \\ B e & \text { Bejan number } \\ k & \text { Thermal conductivity, } \mathrm{W} / \mathrm{m} / \mathrm{K} \\ L & \text { Length, } \mathrm{m} \\ H & \text { Height of cavity, } \mathrm{m} \\ P r & \text { Prandtl number } \\ R a & \text { Rayleigh number based on cavity length } \\ S & \text { Dimensionless entropy generation } \\ T & \text { Temperature, } \mathrm{K} \\ T_{\mathrm{o}} & \text { Bulk temperature }\left(T_{\mathrm{h}}+T_{\mathrm{c}}\right) / 2, \mathrm{~K} \\ u, v & \text { Velocity components in } x \text { and } y \text { directions, } \mathrm{m} / \mathrm{s} \\ U, V & \text { Dimensionless velocity components in } X \text { and } Y \\ & \text { directions } \\ x, y & \text { Dimensional Cartesian coordinates, } \mathrm{m} \\ X, Y & \text { Dimensionless Cartesian coordinates }\end{array}$

$\begin{array}{ll}\text { Greek symbols } \\ \alpha & \text { Thermal diffusivity, } \mathrm{m}^{2} / \mathrm{s} \\ \beta & \text { Thermal expansion coefficient, } \mathrm{K}^{-1} \\ \phi & \text { Irreversibility distribution ratio } \\ v & \text { Kinematic viscosity, } \mathrm{m}^{2} / \mathrm{s} \\ \Omega & \text { Dimensionless vorticity } \\ \theta & \text { Dimensionless temperature } \\ \tau & \text { Dimensionless time } \\ \Psi & \text { Dimensionless stream function } \\ \mu & \text { Viscosity }\left(\mathrm{Ns} / \mathrm{m}^{2}\right)\end{array}$

\section{Subscripts}

av Average

c Cold

f $\quad$ Fluid

f.f Fluid friction

h Hot

h.t Heat transfer

1 Local

t Total

inside a wavy walled enclosure. Numerical solution of flow and temperature fields in a square cavity with heat generation due to an internal heat generating solid body was carried out by Shuja et al. [9]. Recently, the second law thermodynamics was applied to industrial problems to minimize the entropy generation. Yilbas [10] investigated entropy generation of laser short-pulse heating for gold-silver assembly. The entropy generation for laminar convection in a helical coil with a constant heat flux and natural convection from a cylinder with high conductivity fins was handled by Ko [11] and Abu-Hijleh [12], respectively. Naterer et al. [13] presented a review of past advances in numerical analysis using the second law of thermodynamics.

Heat transfer and fluid flow in rectangular cavities are highly influenced by the aspect ratio and Rayleigh number. The aim of the present study is to evaluate the variation of entropy generation with aspect ratio in rectangular cavities having unit dimensionless area but varying aspect ratio from 1 to 16 , Rayleigh number from $10^{2}$ to $10^{5}$ and irreversibility distribution ratio from $10^{-4}$ to $10^{-2}$. The isotherms, streamlines and their corresponding local entropy generation maps are plotted to supply useful information for minimizing the entropy generation in rectangular cavities. The variation of the total entropy generation due to heat transfer and fluid friction irreversibilities and total entropy generation versus aspect ratio are presented in graphical forms. Local and average Bejan numbers are employed to compare the relative importance of heat transfer and fluid friction irreversibilities in the cavity.

\section{The considered problem}

The considered enclosure is schematically illustrated in Fig. 1. It has a length $L$ and height $H$. The horizontal walls are insulated and the vertical walls of the cavity are maintained at different $T_{\mathrm{h}}$ (left wall) and $T_{\mathrm{c}}$ (right wall) constant temperatures such that $T_{\mathrm{h}}>T_{\mathrm{c}}$. The aspect ratio $(\mathrm{AR}=H / L)$ for the problem is changed from 1 to 16 , while the dimensionless area for all cavities is one. The heat transfer in the cavity occurs by laminar natural convection and the effect of radiation is neglected. The problem is solved for air with $\operatorname{Pr}=0.70$.

\section{Mathematical modeling}

The governing equations for the problem are the continuity, momentum and energy equations. The pressure term in the momentum equation can be eliminated using the vorticitystream function approach. By employing the Boussinesq approximation, the dimensionless form of the governing equations can be written as:

$\frac{\partial^{2} \Psi}{\partial X^{2}}+\frac{\partial^{2} \Psi}{\partial Y^{2}}=-\Omega$

$\frac{\partial \Omega}{\partial \tau}+\frac{\partial U \Omega}{\partial X}+\frac{\partial V \Omega}{\partial Y}=\operatorname{Pr}\left(\frac{\partial^{2} \Omega}{\partial X^{2}}+\frac{\partial^{2} \Omega}{\partial Y^{2}}\right)+R a \cdot \operatorname{Pr} \cdot \frac{\partial \theta}{\partial X}$

$\frac{\partial \theta}{\partial \tau}+\frac{\partial U \theta}{\partial X}+\frac{\partial V \theta}{\partial Y}=\frac{\partial^{2} \theta}{\partial X^{2}}+\frac{\partial^{2} \theta}{\partial Y^{2}}$

where $U, V, \theta, \tau, X$ and $Y$ are dimensionless parameters:

$X=\frac{x}{L}, Y=\frac{y}{L}, U=\frac{u}{\alpha L}, V=\frac{v}{\alpha L}, \theta=\frac{\left(T_{\mathrm{f}}-T_{\mathrm{c}}\right)}{\left(T_{\mathrm{h}}-T_{\mathrm{c}}\right)}, \tau=\frac{\alpha t}{L^{2}}$

The dimensionless vorticity, stream function and Rayleigh number are defined as:

$\Omega=\frac{\partial V}{\partial X}-\frac{\partial U}{\partial Y}, U=\frac{\partial \Psi}{\partial Y}, V=-\frac{\partial \Psi}{\partial X}, R a=\frac{g \beta\left(T_{\mathrm{h}}-T_{\mathrm{c}}\right) L^{3}}{\nu \alpha}$ 


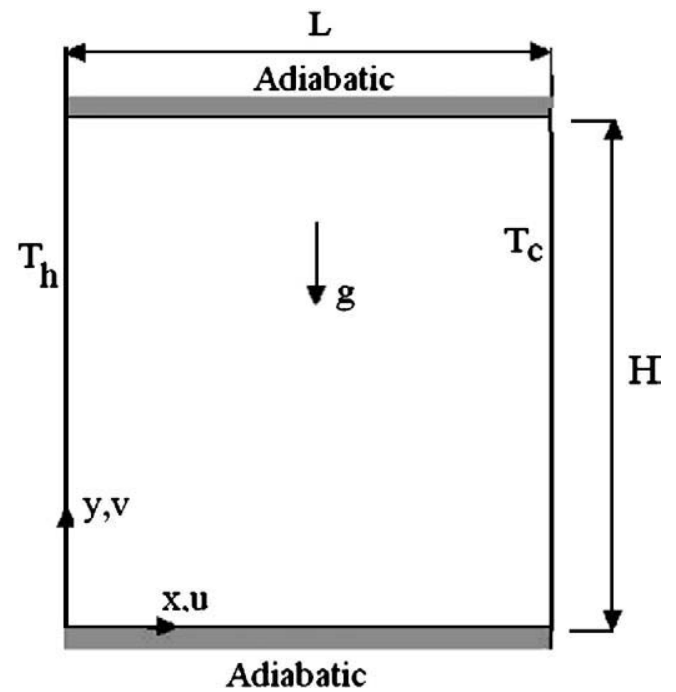

Fig. 1. A schematic view of the considered problem.

The boundary conditions for the problem are:

On the top and bottom walls:

$\Omega=-\frac{\partial U}{\partial Y}, \Psi=0, \frac{\partial \theta}{\partial Y}=0$

On the left and right walls;

$\Omega=\frac{\partial V}{\partial X}, \Psi=0, \theta(0, Y)=0.5, \theta(1, Y)=-0.5$

The corresponding dimensionless initial conditions are:

$U=V=\Psi=\theta=0$ at $\tau=0$

According to the dimensionless parameters defined in Eq. (4) and local thermodynamic equilibrium of the linear transport theory [2], the dimensionless local entropy generation due to heat transfer and fluid friction for a two-dimensional heat and fluid flow in Cartesian coordinates in explicit form can be written as:

$S_{\text {l.h.t }}=\left(\frac{\partial \theta}{\partial X}\right)^{2}+\left(\frac{\partial \theta}{\partial Y}\right)^{2}$

$S_{\text {l.f.f }}=\phi\left\{2\left[\left(\frac{\partial U}{\partial X}\right)^{2}+\left(\frac{\partial V}{\partial Y}\right)^{2}\right]+\left(\frac{\partial U}{\partial Y}+\frac{\partial V}{\partial Y}\right)^{2}\right\}$

The local entropy generation number, which is called the entropy generation number, is the summation of $S_{1 . f . f}$ and $S_{1 . h . t}$ :

$$
\begin{aligned}
S_{1}= & S_{\text {l.t.h }}+S_{\text {l.f.f }}=\left[\left(\frac{\partial \theta}{\partial X}\right)^{2}+\left(\frac{\partial \theta}{\partial Y}\right)^{2}\right] \\
& +\phi\left\{2\left[\left(\frac{\partial U}{\partial X}\right)^{2}+\left(\frac{\partial V}{\partial Y}\right)^{2}\right]+\left(\frac{\partial U}{\partial Y}+\frac{\partial V}{\partial X}\right)^{2}\right\}
\end{aligned}
$$

where $\phi$ is the irreversibility distribution ratio:

$$
\phi=\frac{S_{\text {l.f.f }}}{S_{\text {l.h.t }}}=\frac{\mu T_{\mathrm{o}}}{k}\left(\frac{\alpha}{L \Delta T}\right)^{2}
$$

The total entropy generation is obtained by integrating the dimensionless local entropy generation over the system volume:

$S_{\text {t.h.t }}=\int_{V} S_{\text {l.h.t }} d V, S_{\text {t.f.f }}=\int_{V} S_{\text {l.f.f }} d V, S_{\mathrm{t}}=S_{\text {t.h.t }}+S_{\text {t.f.f }}$

The local Bejan number indicates the strength of the entropy generation due to heat transfer irreversibility [2]:

$B e_{1}=\frac{S_{\text {l.h.t }}}{S_{1}}$

For any location inside the cavity, the condition of $B e_{1}>1 / 2$ implies that the heat transfer irreversibility is dominating, while if $B e_{1}<1 / 2$ the fluid friction irreversibility is dominating. For $B e_{1}=1 / 2$, the heat transfer and viscous irreversibilities are equal. In the present study, the average Bejan number is used to determine the relative importance of the heat transfer irreversibility for the entire cavity:

$B e_{\mathrm{av}}=\frac{\int_{A} B e_{1}(X, Y) \cdot d A}{\int_{A} d A}$

\section{Solution procedure}

The set of governing equations is numerically solved by the finite difference method. The energy and vorticity equations are solved line by line by employing the ADI method, whereas the stream function equation is solved point by point. The finite difference form of diffusion and convection terms are written based on three points central difference which has second order accuracy. The detailed information about the numerical procedure and convergence criteria can be found in Ref. [14].

\subsection{Validation of numerical results}

In order to validate the obtained numerical results, total entropy generation for square cavities with different $\phi$ and $R a$ values were obtained and compared with the results of Magherbi et al. [2]. Fig. 2 depicts the comparison of the present solutions with the reported results. Excellent agreement between the two studies is observed.

\section{Results and discussion}

As was mentioned before, numerical results were obtained for cavities in range of $1<A<16,10^{2}<R a<10^{5}$ and $10^{-4}<\phi<10^{-2}$. Fig. 3 shows the isotherms, streamlines, contours of the local entropy generation due to heat transfer $\left(S_{\text {l.h.t }}\right)$ and fluid friction $\left(S_{\text {l.f.f }}\right)$ and contours of the local entropy generation $\left(S_{1}\right)$ for a cavity with $\mathrm{AR}=1$, $R a=10^{3}$ and $\Phi=10^{-4}$. The isotherms are nearly parallel to the vertical 


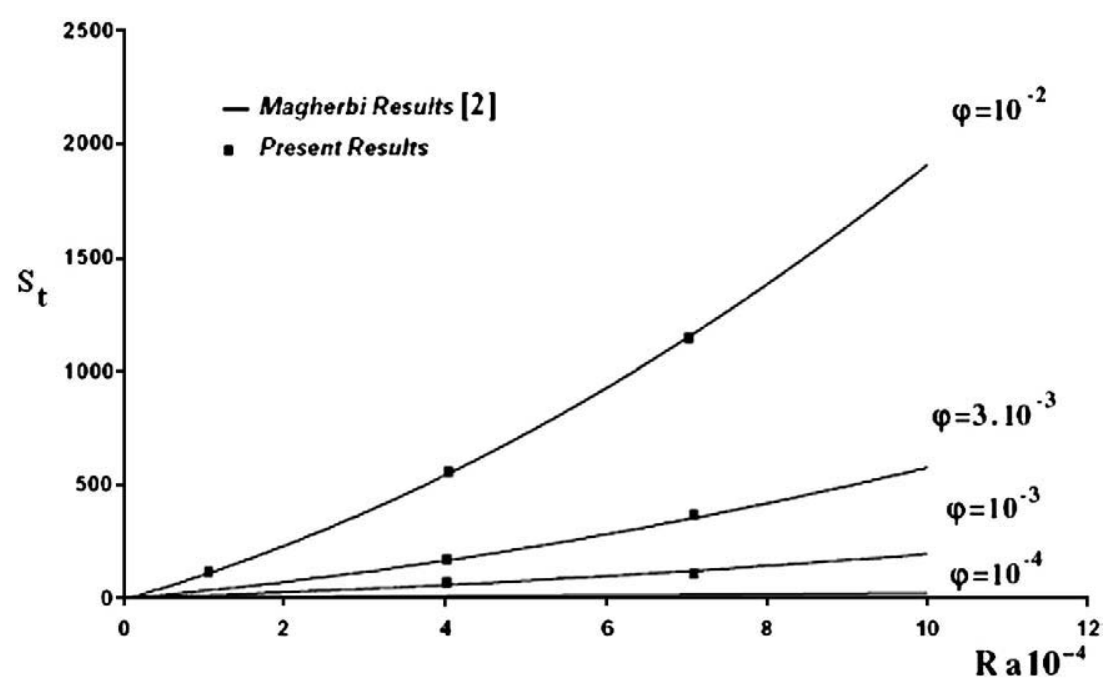

Fig. 2. Comparison of reported numerical solutions with present results.

walls which indicate domination of conduction heat transfer. A comparison of the entropy generation maps for heat transfer (Fig. 3 (c)) and fluid friction (Fig. 3(d)) confirms the less importance of the fluid flow. The maximum local entropy generation due to heat transfer is $\left(S_{\text {l.h.t }}\right)_{\max }=2.27$ while the maximum value of local fluid friction entropy generation is $\left(S_{\text {l.f.f }}\right)_{\max }=0.25$. Hence, the contours of local entropy generation $\left(S_{1}\right)$ are very similar to the local entropy generation due to heat transfer. Fig. 3(f) shows the distribution of the local Bejan number in the cavity. It is observed that the values of contours change from 0.74 to 1 . They are greater than $1 / 2\left(B e_{1}>1 / 2\right)$ which manifests the domination of $S_{1 . h . t}$ in the entire cavity.

The distribution of the same dependent variables as presented in Fig. 3 are shown for a cavity with $R a=10^{5}, \mathrm{AR}=1$ and $\phi=10^{-4}$ in Fig. 4. The enhancement of convection heat transfer in the cavity can be observed by comparison of isotherms, Figs. 3(a) and Fig. 4(a). The isotherms in the cavity are not parallel to the vertical walls due to strong fluid flow. The comparison of $S_{\text {l.h.t }}$ and $S_{\text {l.f.f }}$ maps (Fig. 4(c) and (d)) reveals that the local entropy generation due to fluid friction is considerably greater than the heat transfer local entropy generation. The maximum value of entropy generation due heat transfer is $\left(S_{1 . h .}\right)_{\max }=61.02$ while for fluid friction it is $\left(S_{\text {l.f.f }}\right)_{\max }=563.20$. Hence, the local entropy generation $\left(S_{1}\right)$ map is similar to the fluid friction map $\left(S_{\text {l.f.f }}\right)$. The domination of fluid friction irreversibilities can be seen in Fig. 4(f) which depicts distribution of the local Bejan number in the cavity. The obtained results showed that the local Bejan number varies from 0 to $1,0<B e_{1}<1$.

The isotherms, streamlines, distributions of local heat transfer and fluid friction irreversibilities, local entropy generation and local Bejan number for a tall cavity with $\mathrm{AR}=16, R a=10^{5}$ and $\phi=10^{-4}$ are presented in Fig. 5. In spite of high value of Rayleigh number, the isotherms are parallel to the vertical wall which implies reduction of convection. The comparison of $\left(S_{1 . h . t}\right)_{\max },\left(S_{\text {l.f.f }}\right)_{\max }$ and $\left(\mathrm{S}_{1}\right)_{\max }$ of Fig. 4 with Fig. 5 reveals that the fluid friction irreversibility and local entropy generation decrease with increasing aspect ratio, though a small increase in the value of heat transfer irreversibility is observed.

The variation of the total entropy generation in a cavity with $\Phi=10^{-4}$ versus aspect ratio for different Rayleigh numbers is shown in Fig. 6. The variations of the total entropy generation due to heat transfer $\left(S_{\text {t.h.t }}\right)$ and fluid friction $\left(S_{\text {t.f.f }}\right)$ are illustrated in
Fig. 6(a) and (b), respectively. The changes of the total entropy generation $\left(S_{\mathrm{t}}\right)$ and average Bejan number $\left(B e_{\mathrm{av}}\right)$ with aspect ratio are given in Fig. 6(c) and (d). The total entropy generation due to heat transfer increases with increasing aspect ratio. For low Rayleigh numbers (i.e., $R a=10^{2}, 10^{3}$ ), $S_{\text {t.h.t }}$ almost varies linearly with aspect ratio; though a non-linear behavior appears for high Rayleigh number due to development of the fluid flow. The changes of the total entropy generation due to fluid friction with aspect ratio are completely different. The total entropy generation due to fluid friction rises with increasing aspect ratio, attains a maximum and then it decays. This behavior of $S_{\text {t.f.f }}$ is more obvious for cavities at high values of $R a$ (i.e., $R a=10^{5}$ ). An increase of the aspect ratio improves buoyancy and consequently the fluid flow effects, hence, the values of $S_{\text {t.f.f }}$ increase. However, at a certain value of the aspect ratio, the convection in the cavity becomes weaker and a further increase of the aspect ratio leads to a reduction of $S_{\text {t.f.f. }}$ For a cavity at low values of $R a$, the variation of $S_{\mathrm{t}}$ with aspect ratio is similar to the $S_{\text {t.h.t }}$ variation, though for high values of $R a$, it resembles the $S_{\text {t.f.f }}$ variation. The change of the average Bejan number with aspect ratio is illustrated in Fig. 6 (d). For low Ra numbers (i.e., $R a<10^{4}$ ), the value of the average Bejan number is greater than $1 / 2\left(B e_{\mathrm{av}}>1 / 2\right)$, which shows the strong heat transfer irreversibility in the major part of the cavity. The fluid flow effect improves with increasing Rayleigh number and consequently the value of $B e_{\mathrm{av}}$ decreases. For the cavity with high Rayleigh number (i.e., $R a=10^{5}$ ), $B e_{\mathrm{av}}<1 / 2$ which indicates the domination of fluid friction irreversibilities. In contrast to the variation of the fluid friction irreversibility with aspect ratio, $B e_{\mathrm{av}}$ decays to a minimum value and then rises with increasing aspect ratio.

In order to observe the effect of the irreversibility distribution ratio, the variations of total heat transfer and fluid friction irreversibilities, total entropy generation and average Bejan number for a cavity with $\phi=10^{-2}$ are shown in Fig. 7. The fluid friction local entropy generation varies proportionally with irreversibility ratio (see Eq. (10)). For a cavity with $\phi=10^{-2}$, it is expected that total entropy generation $\left(S_{\mathrm{t}}\right)$ is greatly influenced by $S_{\text {t.f.f }}$ rather than $S_{\text {t.h.t. }}$. The comparison of Fig. 7(a) and (b) reveals the same fact. The values of $S_{\text {t.f.f }}$ are considerably greater than $S_{\text {t.h.t }}$, particularly for high values of the Rayleigh number. Similar to the cavities with $\phi=10^{-4}$, an 


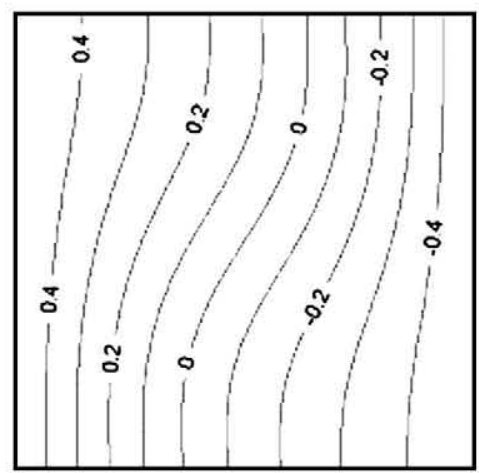

a)

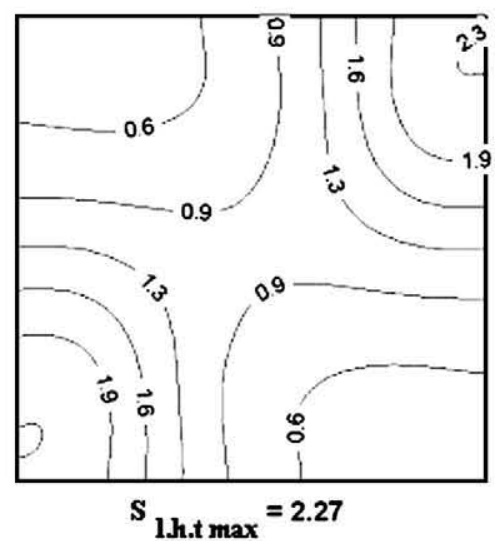

c)

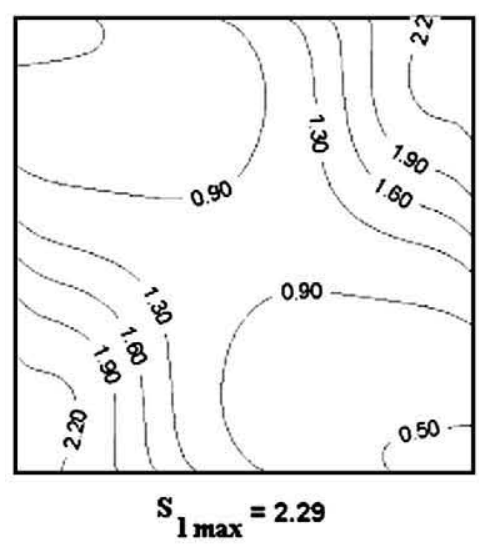

e)

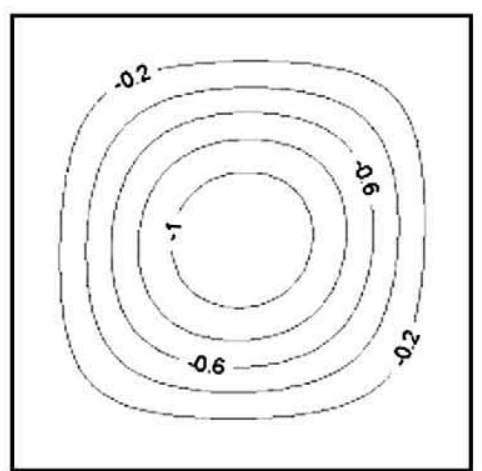

b)

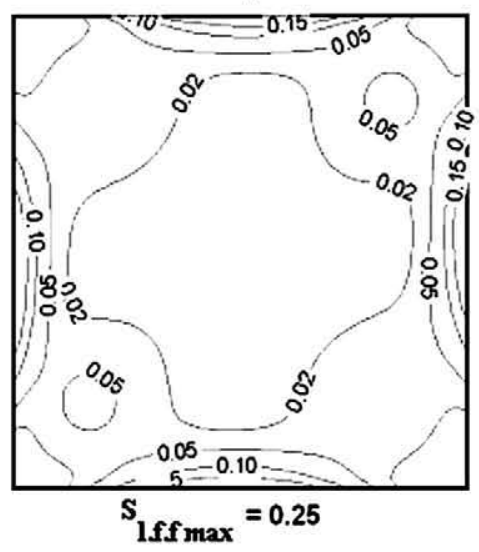

d)

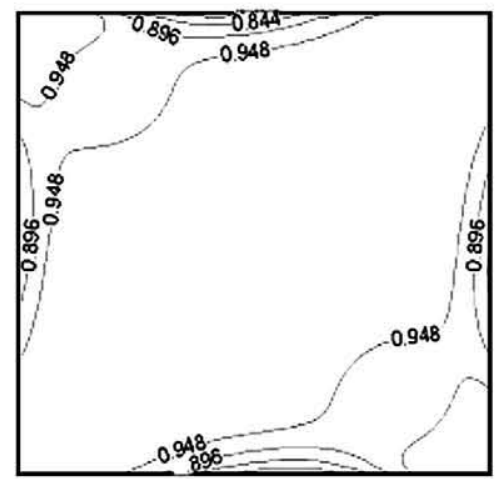

f)

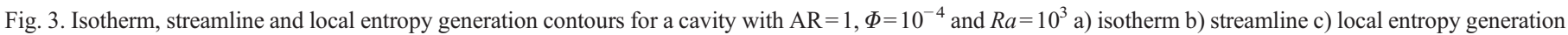
due to heat transfer d) local entropy generation due to fluid friction e) local entropy generation $\mathrm{f}$ ) local Bejan number.

increase of the aspect ratio means that the fluid flow becomes stronger; however convection heat transfer becomes poorer after an aspect ratio. As seen from Fig. 7(d), the variation of the average Bejan number with aspect ratio is different from Fig. 6(d), since the Bejan number is the ratio of $S_{\text {l.h.t }}$ to $S_{\text {l.f.f. An increase of the }}$ irreversibility ratio pulls down the average Bejan number curves since the fluid friction irreversibility is increased.

For all cavities with different aspect ratio, the total entropy generation increases with increasing Rayleigh number, though the rate of increase is different as seen in Fig. 8. For $R a=100$, the total entropy generation increases from the minimum aspect ratio $(\mathrm{AR}=1)$ to the maximum aspect ratio $(\mathrm{AR}=16)$ with the same order. For $R a=10^{5}$ and $\phi=10^{-4}$, the total entropy generation for the $\mathrm{AR}=16$ cavity is less than that for $\mathrm{AR}=9$ due to the peak point of the fluid friction total entropy generation.

\section{Conclusion}

For the rectangular cavity with unit area but different aspect ratio, the variation of local and total heat transfer and 


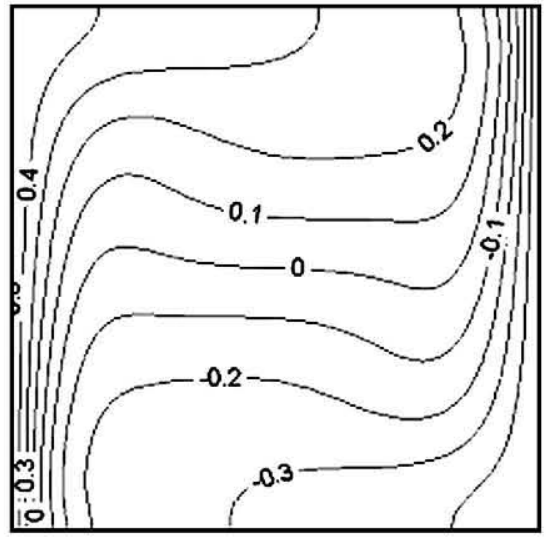

a)

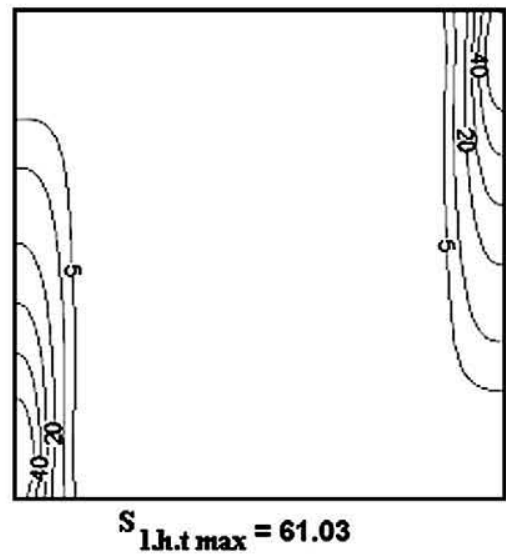

c)

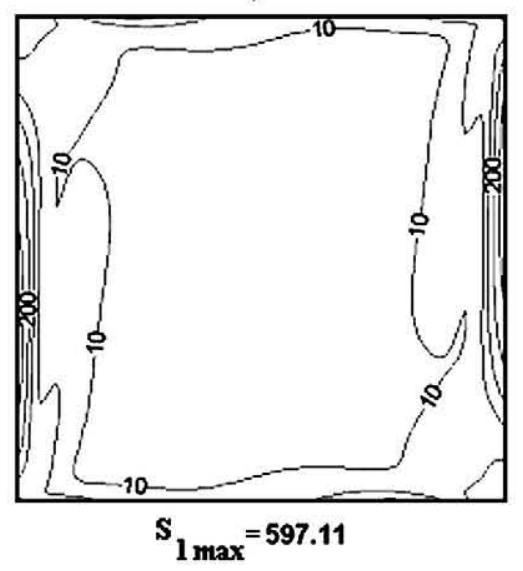

e)

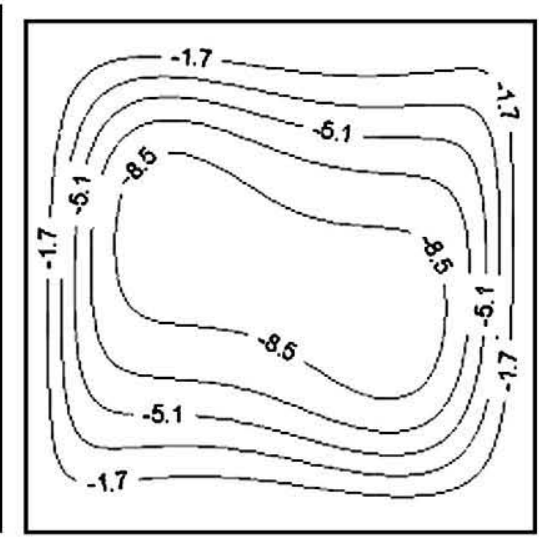

b)

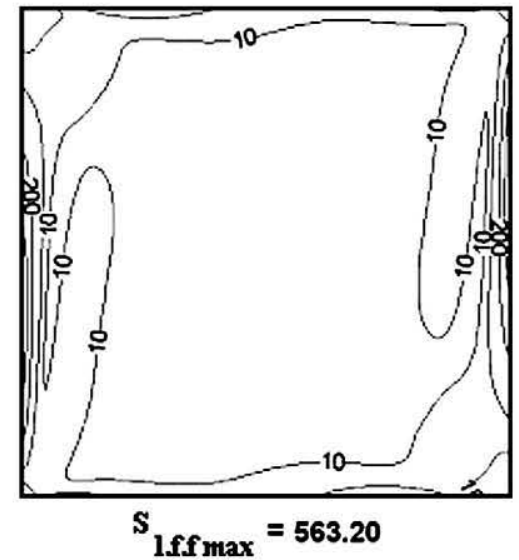

d)

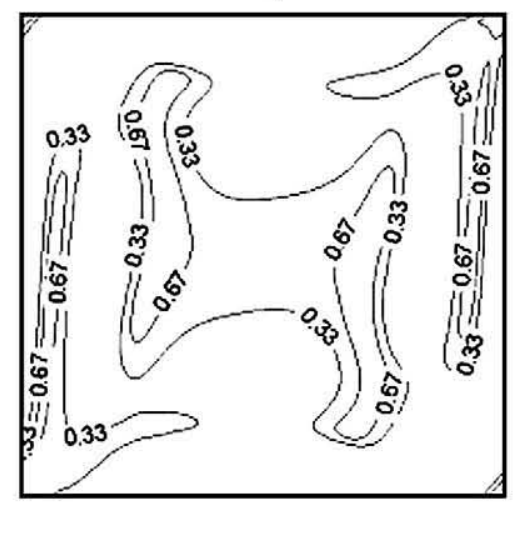

f)

Fig. 4. Isotherm, streamline and entropy generation contours for a cavity with $\mathrm{AR}=1, \Phi=10^{-4}$ and $R a=10^{5}$ a) isotherms b) streamlines c) local entropy generation due to heat transfer d) local entropy generation due to fluid friction e) local entropy generation f) local Bejan number.

fluid friction irreversibilities and entropy generation were investigated. The average Bejan number was used to evaluate the total entropy generation due to heat transfer in the entire domain. Heat transfer and fluid friction irreversibilities in a cavity vary considerably with aspect ratio. For cavities with low $R a$ values (i.e., $R a=10^{2}$ ) and $\varphi=10^{-4}$, the heat transfer irreversibility is strongly dominant and the total entropy generation increases with increase of aspect ratio. For cavities with high $R a$ values (i.e., $R a=10^{5}$ ) and $\varphi=10^{-4}$, fluid friction irreversibility is dominant and total entropy generation increases with aspect ratio, attains a maximum and then it decreases. A peak point for the maximum total entropy generation exists. For cavities with $\varphi=10^{-2}$, the magnitudes of fluid friction irreversibilities are considerably greater than 


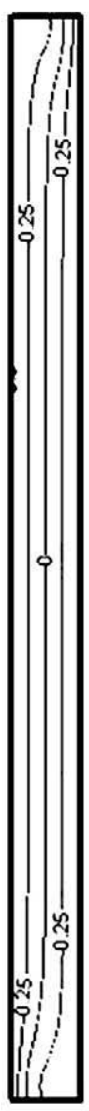

a)

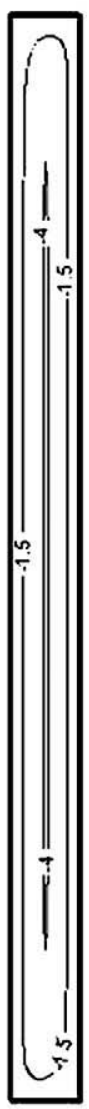

b)

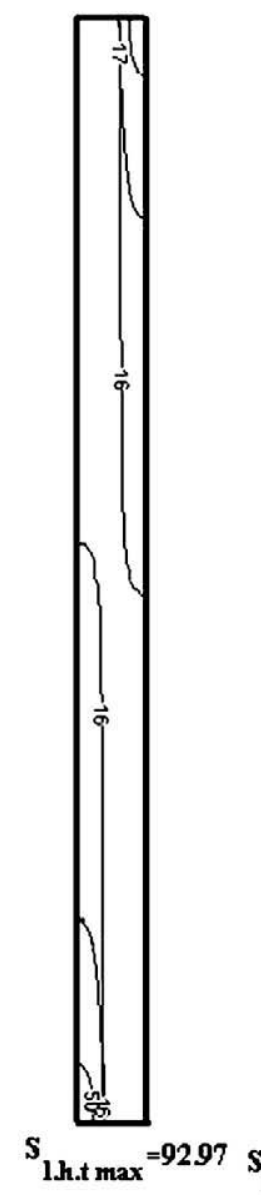

c)

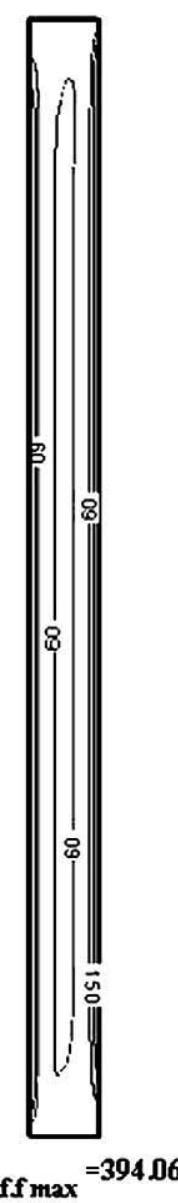

d)

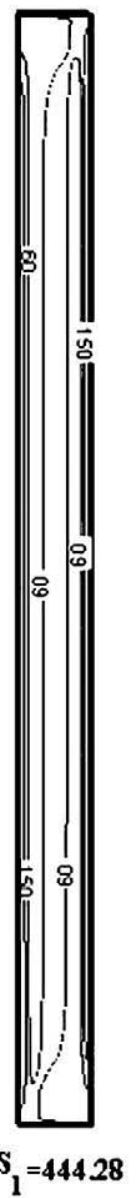

e)

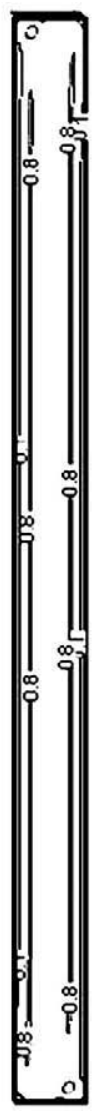

f)

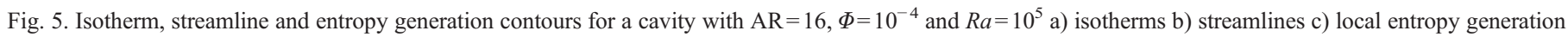
due to heat transfer d) local entropy generation due to fluid friction e) local entropy generation f) local Bejan number.

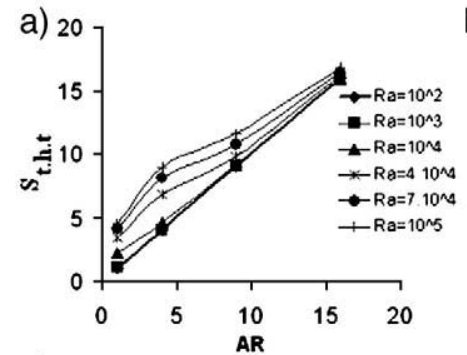

C)

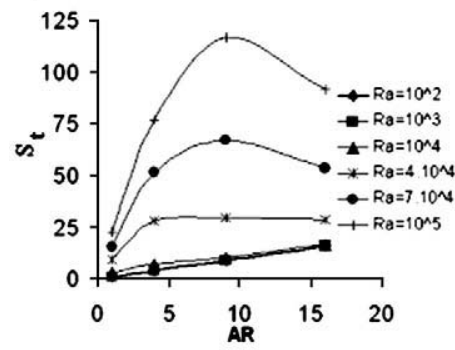

b)

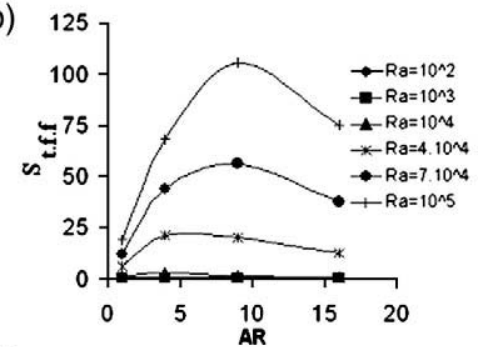

d)

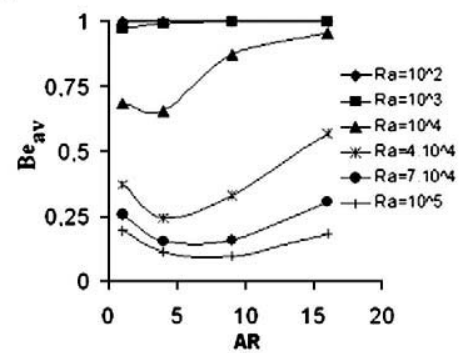

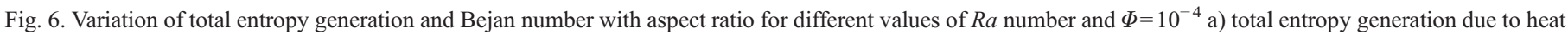
transfer b) total entropy generation due to fluid friction, c) total entropy generation d) average Bejan number. 
a)

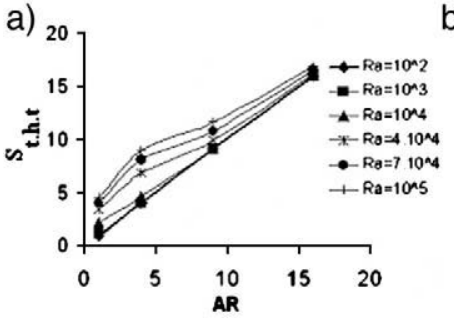

c)

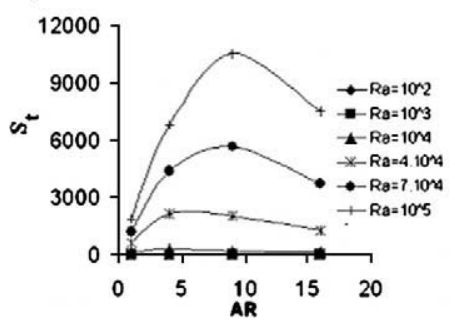

b)

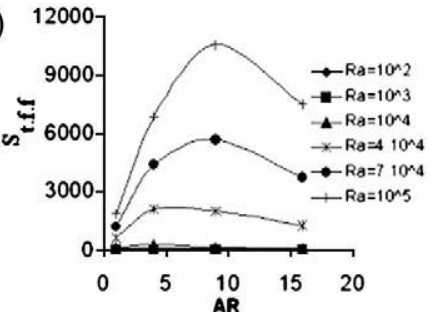

d)

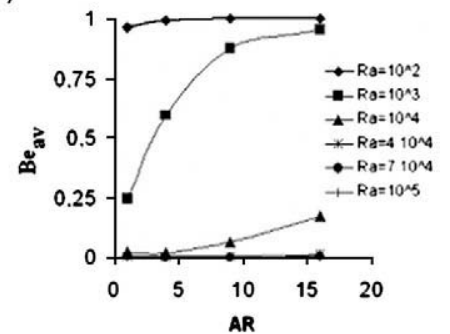

Fig. 7. Variation of total entropy generation and Bejan number with aspect ratio for different values of $R a$ number and $\Phi=10^{-2}$ a) total entropy generation due to heat transfer b) total entropy generation due to fluid friction, c) total entropy generation d) average Bejan number.

for cavities with $\varphi=10^{-4}$. Hence, the fluid friction entropy generation for a cavity with $\varphi=10^{-2}$ is more dominant compared to $\varphi=10^{-4}$. Similar peak points for the total entropy generation were also observed for a cavity with $\varphi=10^{-2}$. The average Bejan number is a proper criterion to predict the domination of heat transfer or fluid friction irreversibilities for the entire domain. The total entropy generation in a cavity increases with Rayleigh number, however, the rate of increase depends on the aspect ratio. For the same Rayleigh number, the total entropy generation for a tall cavity may be less than that for a shorter one.

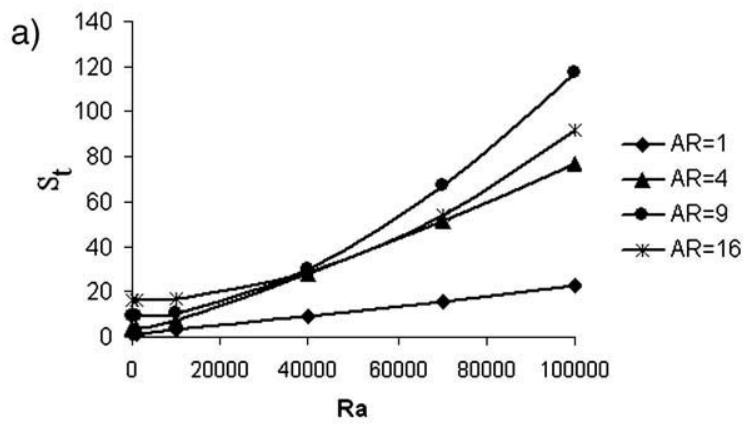

b)

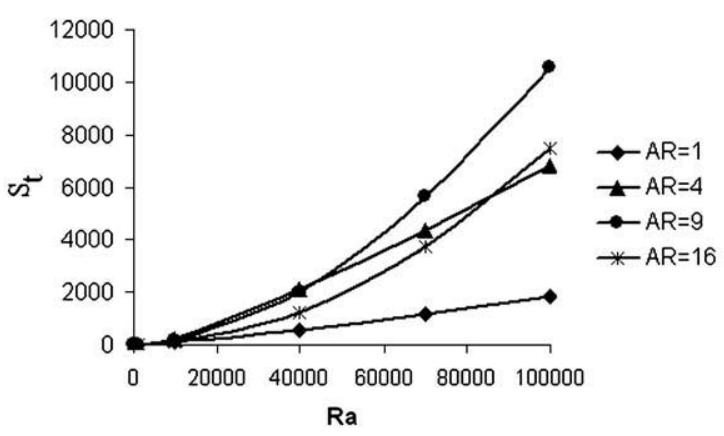

Fig. 8. The changes of total entropy generation with Rayleigh number for cavities with different aspect ratios a) $\Phi=10^{-4}$, b) $\Phi=10^{-2}$.

\section{References}

[1] I. Dagtekin, H.F. Oztop, A. Bahloul, Entropy generation for natural convection in $\Gamma$-shaped enclosures, Int. Commun. Heat Mass Transf. 34 (2007) 502-510.

[2] M.A. Magherbi, A. Abbassi, A.B. Brahim, Entropy generation at the onset of natural convection, Int. J. Heat Mass Transfer 46 (2003) 3441-3450.

[3] B.S. Yilbas, S.Z. Shuja, S.A. Gbadebo, H.I. Abu Al-Hamayel, K. Boran, Natural convection and entropy generation in a square cavity, Int. J. Energy Res. 22 (1998) 1275-1290.

[4] L.B. Erbay, Z. Altac, B. Sulus, An analysis of the entropy generation in a square enclosure, Entropy 5 (2003) 496-505.

[5] L.B. Erbay, Z. Altac, B. Sulus, Entropy generation in a square enclosure with partial heating from a vertical lateral wall, Heat Mass Transf. 40 (2004) 909-918.

[6] A.C. Baytas, W.J. Yang, A. Arbor, Optimization in an inclined enclosure for minimum entropy generation in natural convection, J. Non-Equilib. Thermodyn. 22 (1997) 145-155.

[7] A. Andreozzi, A. Auletta, O. Manca, Entropy generation in natural convection in a symmetrically and uniformly heated vertical channel, Int. J. Heat Mass Transfer 49 (2006) 3221-3228.

[8] S. Mahmud, A.K.M. Sadrul Islam, Laminar free convection and entropy generation inside an inclined wavy enclosure, Int. J. Therm. Sci. 42 (2003) 1003-1012.

[9] S.Z. Shuja, B.S. Yilbas, M.O. Budair, Natural convection in a square cavity with a heat generating body: entropy consideration, Heat Mass Transf. 36 (2000) 343-350.

[10] B.S. Yilbas, Laser short-pulse of gold-silver assembly: entropy generation due to heat and electricity flows in electron subsystem, Numer. Heat Transf. A 49 (2006) 873-891.

[11] T.H. Ko, Numerical investigation of laminar forced convection and entropy generation in a helical coil with constant wall heat flux, Numerical Heat Transfer Part A 49 (2006) 257-278.

[12] B. Abu-Hijleh, Natural convection and entropy generation from a cylinder with high conductivity fins, Numer. Heat Transf. A 39 (2001) 405-432.

[13] G.F. Naterer, J.A. Camberos, Entropy and the second law fluid flow and heat transfer simulation, J. Thermophys. Heat Transf. 17 (2003) 360-371.

[14] M. Mobedi, Conjugate natural convection heat transfer in a square cavity with finite thickness horizontal walls, Int. Comm. Heat Mass Transfer 35 (2008) 503-513. 